\title{
Capacidad fitorremediadora de Hydrocotyle ranunculoides L. f., en aguas contaminadas con mercurio
}

\section{Phytoremediation capacity of Hydrocotyle ranunculoides L. f., in waters contaminated with mercury}

\author{
Abigail Rumaja ${ }^{1}$, Jackeline Z. Huamán ${ }^{1}$ \& Anahí Cardona ${ }^{1}$ \\ ${ }^{1}$ Universidad Nacional de San Antonio Abad del Cusco \\ Correo electronico:anahi.cardona@unsaac.edu.pe
}

Resumen

El objetivo fue evaluar la capacidad fitorremediadora de la especie vegetal $H$. ranunculoides $L$. f., en relación a la contaminación de muestras de agua con mercurio a diferentes concentraciones. Para lo cual se realizaron tres etapas, la primera fue la recolección y el cultivo en sistema hidropónico, la segunda la contaminación con mercurio (II) en muestras de agua en laboratorio y la tercera evaluar la capacidad fitorremediadora. Para el análisis del mercurio se usó la técnica de Espectrofotometría de Absorción Atómica (AAS). También se evaluó las alteraciones morfológicas y de composición. La exposición de la especie vegetal a la contaminación con mercurio a las concentraciones de $0.1,0.5,1.0,2.0$ y 5.0 ppm duró 15 días. En los resultados, muestran que la concentración $1.0 \mathrm{ppm}$ genera clorosis y a $5.0 \mathrm{ppm}$ necrosis; en cuanto a la longitud de raíz, tallo y hojas se observó disminución proporcional a la concentración de mercurio. La morfología interna muestra alteraciones por el mercurio a nivel de pared celular, organización de tejidos y sistema vascular, tanto en raíz, tallo y hoja. En la composición se observó disminución del porcentaje de humedad, cenizas, proteínas, grasas y fibra e incremento del porcentaje de carbohidratos, en diferentes concentraciones. El factor de bioacumulación fue mayor a $1000 \mathrm{mg} / \mathrm{L}$ hasta la concentración $1.0 \mathrm{ppm}$ de mercurio. El factor de traslocación en las concentraciones $0.1 \mathrm{y}$ $0.5 \mathrm{ppm} \mathrm{Hg} 2+$ es $>1 \mathrm{mg} / \mathrm{L}$ y en concentraciones de $1.0,2.0$ y $5.0 \mathrm{ppm} \mathrm{Hg} 2+$ el mercurio se encuentra en mayor proporción en raíz.

Palabras clave: Fitorremediación; mercurio; factor de bioacumulación (BAF); factor de traslocación (FT); Hydrocotyle ranunculoides L. f. espectrofotometría de absorción atómica.
Abstract
The objective was to evaluate the phytoremediation capacity of the plant species H. ranunculoides L. f., In relation to the contamination of water samples with mercury at different concentrations. For which three stages 
were carried out, the first was the collection and cultivation in a hydroponic system, the second the contamination with mercury (II) in laboratory water samples and the third evaluated the phytoremediation capacity. For the analysis of mercury, the Atomic Absorption Spectrophotometry (AAS) technique was used. Morphological and compositional alterations were also evaluated. Exposure of the plant species to mercury contamination at concentrations of $0.1,0.5,1.0,2.0$, and $5.0 \mathrm{ppm}$ lasted 15 days. In the results, they show that the $1.0 \mathrm{ppm}$ concentration generates chlorosis and at 5.0 ppm necrosis; As for the length of the root, stem and leaves, a proportional decrease was observed in the mercury concentration. Internal morphology shows alterations by mercury at the cell wall level, tissue organization and vascular system, both in root, stem and leaf. In the composition, a decrease in the percentage of humidity, ashes, proteins, fats and fiber and an increase in the percentage of carbohydrates were observed, in different concentrations. The bioaccumulation factor was greater than $1000 \mathrm{mg} / \mathrm{L}$ up to the $1.0 \mathrm{ppm}$ concentration of mercury. The translocation factor in concentrations 0.1 and $0.5 \mathrm{ppm} \mathrm{Hg} 2+$ is $>1 \mathrm{mg} / \mathrm{L}$ and in concentrations of 1.0, 2.0 and $5.0 \mathrm{ppm} \mathrm{Hg} 2+$ mercury is found in a higher proportion in the root.

Keywords: Phytoremediation; mercury; bioaccumulation factor (BAF); factor of traslocation (FT); atomic absorption spectrophotometry.

\section{Introducción}

La fitorremediación tiene como mecanismo la bioacumulación, que consiste básicamente en absorber, concentrar y precipitar metales pesados en el interior de las células de las especies vegetales. Estos procesos se pueden dar en diferentes partes de la planta, como son vacuolas y pared celular de hojas, tallos y raíces (Cabo \& Frers, 2008; Delgadillo et al, 2011) Para realizar este estudio se tomó en cuenta la problemática de la alta contaminación del agua por metales pesados entre ellos el mercurio, cuya fuente son los residuos de actividades productivas como: la minería, industria, agricultura (fertilizantes y herbicidas), y también debido a causas naturales por fenómenos geológicos (meteorización, la erosión de las rocas y la lixiviación). Por tal motivo se buscan nuevas técnicas de descontaminación, una de estas sería la fitorremediación con macrófitas acuáticas como es Hydrocotyle ranunculoides (Mateccllo) (Pinto, 2014; Cubero \& Vieira, 1999).

Los recursos hídricos a nivel mundial vienen siendo afectados principalmente por la actividad minera en la cual se lleva acabo procesos de lixiviación provocando la liberación de altas concentraciones de metales pesados entre ellos el mercurio (Díaz, 2016). El mercurio debido a su alta toxicidad puede ocasionar efectos deletéreos en la salud. Se habla además de que dicho metal es bioacumulable, permaneciendo entre 18 y 30 años en el organismo, también compite con el Fe, $\mathrm{Zn}, \mathrm{Mn}$, Se, y $\mathrm{Cu}$ por los ligandos en sistemas biológicos alterando funciones metabólicas. Los metales se incorporan usualmente a las proteínas, algunas de las cuales funcionan como enzimas o catalizadores biológicos (Pinto, 2014; Gisbert, 2001). 
En Cusco, Perú, crece la especie vegetal Hydrocotyle ranunculoides (Mateccllo); que de comprobarse su capacidad fitorremediadora en relación a la contaminación con mercurio, podría ser utilizada en el tratamiento de aguas contaminadas por este metal, mediante la construcción de humedales artificiales especialmente en zonas afectadas por la actividad minera.

El presente trabajo de investigación tiene por objetivo evaluar la capacidad fitorremediadora de la especie vegetal Hydrocotyle ranunculoides (Mateccllo) en relación a la contaminación con mercurio a diferentes concentraciones.

\section{Metodología}

\section{Materiales y métodos}

\section{Lugar de ejecución del trabajo.}

El cultivo hidropónico se llevó a cabo en un gabinete adecuado para la adaptación de las especies vegetales, ubicado a una altitud de 3235 m.s.n.m. en la ciudad de Cusco, Perú. La etapa de contaminación con mercurio, preparación de reactivos y análisis por espectrofotometría de masas se realizó en el laboratorio MCQUIMICALAB Cusco, Perú.

El procesamiento y lectura de las muestras se realizó en el laboratorio de la Escuela Profesional de Ingeniería Química de la Universidad Nacional San Antonio Abad del Cusco, Perú. El análisis proximal de la especie vegetal y preparación de reactivos se realizó en el laboratorio de Química de la Escuela Profesional de Química de la Universidad Nacional San Antonio Abad del Cusco, Perú.

El análisis de la morfología interna y externa de la especie vegetal se realizó en el laboratorio de Farmacognosia y Productos Naturales de la Escuela Profesional de Farmacia y Bioquímica de la Universidad Nacional de San Antonio Abad del Cusco, Perú.

\section{Diseño del estudio.}

Se realizó un estudio de tipo cuasi experimental. Se usó un análisis secundario de una investigación primaria (Huamán y Abigail, 2017).

Procedimiento para determinar la concentración de mercurio en raíz, parte aérea y agua.

Método de la ditizona - $3500 \mathrm{Hg}$ C. (Eaton, Cresceri y Greenberg, 1995) (Sandell, 1950). Fundamento de la extracción de mercurio por el método de la ditizona

El mercurio ionizado reacciona con la ditizona para dar un omplejo coloreado: el ditizonato de mercurio, que presenta tonos entre anaranjado y rojo. La ditizona disminuye la tensión superficial de las disoluciones, produciendo gotas pequeñas y aumentando la cantidad de muestra que llega a la llama. La ditizona es el reactivo de elección cuando es cuestión de aislar mercurio de una solución conteniendo una sola parte pequeña de metales pesados tales 
como cobre, plata, zinc, etc., porque al combinarse la ditizona y el mercurio forma ditizonato de mercurio, el cual puede ser extraído cuantitativamente de la solución débilmente básica, esta es fácilmente descompuesta por agitación del solvente orgánico con una solución ácida diluida $0.01 \mathrm{~N}$ de ácido sulfúrico.

\section{Determinación del factor de bioacumualción (BAF) y factor de traslocación (Mendieta W. C. \& Taisigue L. K., 2014)}

El cálculo del factor de bioacumulación (BAF) se hizo para estimar la relación entre los residuos de mercurio en las plantas y las concentraciones medidas en el medio donde viven (agua). Los BAF se calcularon dividiendo la concentración de mercurio total en la planta con la del agua. Los factores de bioacumulación indican qué especies de plantas pueden ser consideradas acumuladoras, tolerantes o exclusoras.

El factor de traslocación se obtuvo dividiendo la concentración en el órgano de interés (parte aérea) entre la concentración en la raíz. Los factores de bioacumulación y traslocación indicarán qué especies de plantas pueden ser consideradas acumuladoras, tolerantes o exclusoras.

Las plantas con $\mathrm{BAF} \geq 1000 \mathrm{mg} / \mathrm{L}$ y $\mathrm{FT} \geq 1 \mathrm{mg} / \mathrm{L}$ son considerados como acumuladoras o híper acumuladoras, las que presentan valores $<1000 \mathrm{mg} / \mathrm{L}$ y $<1 \mathrm{mg} / \mathrm{L}$, respectivamente, son consideradas como tolerantes y las que presentan valores $<100$ $\mathrm{mg} / \mathrm{L} \mathrm{y}<1 \mathrm{mg} / \mathrm{L}$ se consideran como plantas exclusoras.

Fórmula del factor de bioacumulación (BAF):

$$
B A F=\frac{[\mathrm{Hg}] \text { tejidos }}{[\mathrm{Hg}] \text { agua }}
$$

Donde:

$[\mathrm{Hg}]$ tejidos= Es la concentración total de mercurio en el tejido vegetal (raíz y parte aérea).

$[\mathrm{Hg}]$ agua $=$ Es la concentración de mercurio inicial en el agua contaminada

Fórmula del factor de traslocación (FT):

$$
F T=\frac{([\mathrm{Hg}] \text { parte aérea })}{[\mathrm{Hg}] \text { raíz }}
$$

Donde:

$[\mathrm{Hg}]$ parte aérea $=$ Es la concentración total de mercurio en parte aérea.

$[\mathrm{Hg}]$ agua $=$ Es la concentración total de mercurio en raíz. 


\section{Procedimiento general para la caracterización de la morfología externa e interna de la especie vegetal Hydrocotyle ranunculoides}

- Evaluación de la presencia de clorosis y necrosis en Hydrocotyle ranunculoides durante la contaminación con mercurio (Azcón \& Talón, 2008) Durante los 15 días de contaminación se observó la aparición de clorosis y necrosis foliar a las diferentes concentraciones de mercurio.

- Descripción morfológica externa (morfometría) (Azcón \& Talón, 2008) La morfometría se realizó antes y después de la contaminación del agua con mercurio, donde después de la contaminación que duró 15 días se observaron cambios en el crecimiento tanto de la raíz como el de la parte aérea. Para obtener las características morfológicas de las muestras vegetales, se utilizó el vernier y la observación directa, considerando los siguientes parámetros: Talla de la planta en cm.

Longitud del tallo en $\mathrm{cm}$.

Diámetro del tallo en $\mathrm{cm}$.

Longitud de raíz en $\mathrm{cm}$.

Longitud de hojas en $\mathrm{cm}$.

Ancho de hojas en cm

- Descripción morfológica interna (Azcón \& Talón, 2008)

Después de los 15 días de contaminación con mercurio se procedió a realizar los cortes histológicos de ambas especies para observar los efectos tóxicos de este metal a nivel de tejidos.

- Índice estomático y densidad estomática (Barrientos \& Borys, 2003)

Índice estomático:

$$
I E=\frac{(\text { Número de estomas) }}{\text { (Número de células epidérmicas }+ \text { número de estomas) }} \times 100
$$

- Densidad estomática

$$
D E=\frac{\text { (Número de estomas) }}{m m^{2}}
$$

Representa el cociente entre el número de estomas y la cantidad de células epidérmicas.

\section{Procedimiento para el análisis proximal}

Este procedimiento se realizó antes y después de la contaminación con mercurio, con la finalidad de determinar las concentraciones de grasas, proteínas y carbohidratos ya que estos tienen un papel importante en el mecanismo de fitorremediación.

- Determinación de proteína total (Método kjeldahl AOAC, 991.20) (Adaptado) (AOAC International, Official methods of analysis of AOAC International., S. F.) 
Fundamento: Toda materia orgánica es degradable cuando se somete a un proceso de digestión en presencia de un ácido y catalizador formándose sales que contienen nitrógeno, el nitrógeno se libera en forma de amoniaco por una reacción de sustitución con una base fuerte, el nitrógeno liberado se captura en ácido para luego cuantificar por titulación ácido-base.

Cálculos:

$$
\% N_{\text {total }}=\frac{\left(V_{f}\right) \times N_{H 2 S O 4} \times 0.014}{m} \times 100
$$

Donde:

$\% \mathrm{~N}$ total: Porcentaje de nitrógeno total

$\mathrm{N}=$ Normalidad de solución estándar de $\mathrm{H} 2 \mathrm{SO} 4$

$\mathrm{Vf}=$ Volumen final de $\mathrm{H} 2 \mathrm{SO} 4$ consumido

$\mathrm{m}=$ Peso en $\mathrm{g}$ de la muestra

Para obtener la cantidad de proteína bruta se multiplica por el factor de conversión que es 6.25 para productos vegetales.

- Determinación de fibra total (Método gravimétrico hidrólisis ácida y alcalina AOAC. 920,86) (Adaptado) (AOAC International, Official methods of analysis of AOAC International., S. F.)

Fundamento: Su determinación se basa en la simulación de la digestión en el organismo por compuestos ácidos y alcalinos separando los constituyentes solubles de los insolubles.

Cálculos:

Donde:

$$
\% \text { Fibra total }=\frac{p_{1}-p_{2}}{p_{m}} \times 100
$$

$\mathrm{P} 1=$ Peso de la fibra cruda después de la digestión ácida alcalina en $(\mathrm{g})$

$\mathrm{P} 2=$ Peso de la muestra incinerada (ceniza), (g).

$\mathrm{Pm}=$ Peso de la muestra $(\mathrm{g})$

- Determinación de ceniza total (Método gravimétrico, por calcinación AOAC 940.26 (Adaptado) (AOAC International, Official methods of analysis of AOAC International., S. F.)

Fundamento: El agua y los vapores son volatilizados y la materia orgánica es quemada en presencia de oxígeno en aire a $\mathrm{CO} 2$ y óxido de nitrógeno, la mayoría de los minerales son convertidos, en óxidos, sulfatos, fosfatos, cloruros y silicatos, elementos como el hierro, selenio, plomo y mercurio, pueden volatilizarse parcialmente con este procedimiento. El porcentaje de ceniza se determina sometiendo a calcinación por 2 horas. A una temperatura de $600{ }^{\circ} \mathrm{C}$ hasta la eliminación de materia orgánica y quedando solo óxidos metálicos presentes y se calcula con la siguiente fórmula: 


$$
\% \text { Ceniza total }=\frac{M_{2}-M_{1}}{M} \times 100
$$

- Determinación de grasas totales (Método gravimétrico, extracción con Socklet AOAC) (AOAC International, Official methods of analysis of AOAC International., S. F.) Fundamento: Todos los lípidos tienen la propiedad común de ser solubles en solventes orgánicos (metanol, etanol, acetona, cloroformo, éter, hexano, benceno, etc.) e insolubles en agua.

Cálculos:

$$
\% \text { Grasa total }=\frac{P 2-P 1}{M} \times 100
$$

Donde:

$\mathrm{M}=$ Peso de la muestra seca en gramos.

$\mathrm{P} 2=$ Peso del balón en gramos.

$\mathrm{P} 1=$ Peso del balón más grasa en gramos.

- Determinación de carbohidratos totales (Cálculo por diferencia) (Cano, 1999, Juárez, 2011)

Llamados también glúcidos, hidratos de carbono o sacáridos, son moléculas orgánicas compuestas por carbono, hidrógeno y oxígeno. Son solubles en agua. Se calculó por diferencia; restando de 100 los porcentajes de humedad, proteínas, grasas, y cenizas como se muestra en la fórmula.

$$
\text { \% C.H. }=100-(\% \text { Humedad }+\% \text { Grasa }+\% \text { Proteína }+\% \text { Ceniza })
$$

\section{Resultados}

Determinación de la concentración final de mercurio en raíz y parte aérea (hojas y tallos); de la especie vegetal Hydrocotyle ranunculoides

En la tabla 1, se muestran las concentraciones de mercurio absorbidas por la especie vegetal $H$. ranunculoides, en estas se observa que a bajas concentraciones existe una mayor traslocación de la raíz a la parte aérea y a altas concentraciones disminuye la traslocación de mercurio e incrementa la acumulación en raíz. 


\section{Tabla 1}

Concentración final de mercurio

Hydrocotyle ranunculoides (Mateccllo)

\begin{tabular}{|c|c|c|c|}
\hline $\begin{array}{c}\text { Parte } \\
\text { vegetal }\end{array}$ & $\begin{array}{l}\text { Concentración de } \\
\text { mercurio (ppm) }\end{array}$ & Número de muestras & $\begin{array}{c}{ }^{*} \text { Media } /{ }^{* *} \text { DS } \\
\text { Concentración de mercurio } \\
(\mathrm{ppm})\end{array}$ \\
\hline \multirow{7}{*}{ 请 } & Control & 3 & $0.000 / \pm 0.000$ \\
\hline & 0.1 & 3 & $0.017 / \pm 0.0056$ \\
\hline & 0.5 & 3 & $0.104 / \pm 0.0036$ \\
\hline & 1 & 3 & $0.167 / \pm 0.0087$ \\
\hline & 2 & 3 & $0.265 / \pm 0.0042$ \\
\hline & 5 & 3 & $0.371 / \pm 0.0035$ \\
\hline & Control & 3 & $0.000 / \pm 0.000$ \\
\hline \multirow{5}{*}{ 逫 } & 0.1 & 3 & $0.024 / \pm 0.0061$ \\
\hline & 0.5 & 3 & $0.090 / \pm 0.0035$ \\
\hline & 1 & 3 & $0.111 / \pm 0.0089$ \\
\hline & 2 & 3 & $0.207 / \pm 0.0038$ \\
\hline & 5 & 3 & $0.272 / \pm 0.0030$ \\
\hline
\end{tabular}

DS: Desviación estándar

\section{Determinación de la concentración final de mercurio en agua}

En la tabla 2, se muestran los resultados de la concentración final de mercurio en agua y el porcentaje de remoción, donde se observa una disminución frente a las concentraciones iniciales, lo cual nos indica que a menor concentración de mercurio en agua hay mayor porcentaje de remoción y va disminuyendo a altas concentraciones. 
Tabla 2

Concentración de mercurio en agua

Grupo de prueba positivo: Hydrocotyle ranunculoides (Mateccllo)

\begin{tabular}{|c|c|c|c|c|}
\hline \multirow{2}{*}{$\begin{array}{c}\text { Concentración de } \\
\text { mercurio (ppm) }\end{array}$} & \multirow{2}{*}{$\begin{array}{c}\text { Número de } \\
\text { muestras }\end{array}$} & \multicolumn{2}{|c|}{$\begin{array}{c}\text { *Media /** DS } \\
\text { Concentración de mercurio } \\
\text { (ppm) }\end{array}$} & \multirow[t]{2}{*}{$\%$ de remoción } \\
\hline & & 0 & \pm 0.000 & \\
\hline 0.1 & 3 & 0.0033 & \pm 0.0012 & 97 \\
\hline 0.5 & 3 & 0.0483 & \pm 0.0029 & 90.4 \\
\hline 1 & 3 & 0.3513 & \pm 0.0025 & 64.9 \\
\hline 2 & 3 & 0.9 & \pm 0.0010 & 55 \\
\hline 5 & 3 & 3.499 & \pm 0.0017 & 30.02 \\
\hline \multicolumn{5}{|c|}{ Grupo de prueba negativo: sin especie vegetal } \\
\hline $\begin{array}{l}\text { Concentración de } \\
\text { mercurio (ppm) }\end{array}$ & $\begin{array}{l}\text { Número de } \\
\text { muestras }\end{array}$ & \multicolumn{3}{|c|}{ Resultado de concentración de mercurio (ppm) } \\
\hline CONTROL 0.1 & 1 & \multicolumn{3}{|c|}{0.1} \\
\hline CONTROL 0.5 & 1 & \multicolumn{3}{|c|}{0.45} \\
\hline CONTROL 1.0 & 1 & \multicolumn{3}{|c|}{0.9} \\
\hline CONTROL 2.0 & 1 & \multicolumn{3}{|c|}{1.979} \\
\hline CONTROL 5.0 & 1 & \multicolumn{3}{|c|}{4.93} \\
\hline
\end{tabular}

Nota:* Promedio de 3 muestras ** Desviación Estándar

Determinación del factor de bioacumulación (BAF) y factor de traslocación (FT) de la especie vegetal Hydrocotyle ranunculoides

En la tabla 3, se observan los valores de BAF para $H$. ranunculoides a concentraciones: $0.1,0.5$ y $1.0 \mathrm{ppm}$ de $\mathrm{Hg} 2+$ el BAF es $>1000 \mathrm{mg} / \mathrm{L}$. Por lo que podemos decir que a concentraciones bajas (0.1-1.0 ppm de $\mathrm{Hg} 2+$ ) acumula mercurio tanto en raíz como en parte aérea, y a medida que incrementa la concentración del contaminante disminuye la acumulación en la parte aérea.

Tabla 3

Factor de bioacumulación de traslocación

Hydrocotyle ranunculoides

\begin{tabular}{ccc}
\hline & BAF & FACTOR DE TRASLOCACION \\
\cline { 2 - 3 } $\begin{array}{c}\text { CONCENTRACION } \\
\text { DE MERCURIO } \\
\text { (ppm) }\end{array}$ & Concentración de mercurio (ppm) & Concentración de mercurio (ppm) \\
\hline CONTROL & $0.00 / \pm 0.000$ & $0.00 / \neq 0.000$ \\
\hline 0.1 & $3761.09 / \pm 0.069$ & $1.56 / \neq 0.080$ \\
0.5 & $1961.22 / \pm 0.003$ & $1.05 / \neq 0.004$ \\
1 & $1332.66 / \pm 0.060$ & $0.76 / \neq 0.009$ \\
2 & $874.93 / \neq 0.010$ & $0.78 / \neq 0.030$ \\
5 & $295.50 / \pm 0.000$ & $0.73 / \neq 0.010$ \\
\hline
\end{tabular}

* Promedio de 3 muestras ${ }^{\star \star}$ Desviación Estándar 
Caracterización de la morfología interna raíz y parte aérea (tallos y hojas) de la especie vegetal Hydrocotyle ranunculoides después de la contaminación con mercurio, mediante cortes histologicos

En la figura 1, 2 y 3 se observa los cortes transversales realizados en la hoja, tallo y raíz.

\section{Figura 1}

Corte transversal de hoja del Grupo Control

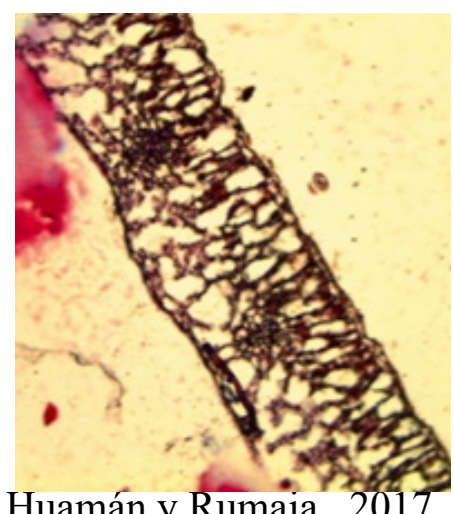

Fuente: Huamán y Rumaja, 2017

\section{Figura 2}

Corte transversal de tallo Grupo control

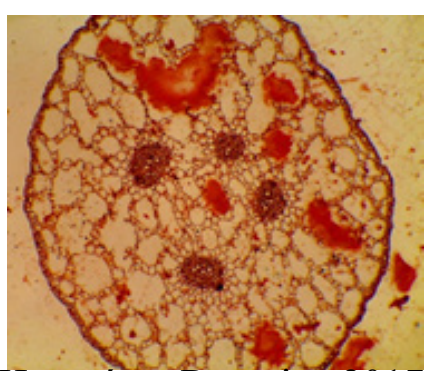

Fuente: Huamán y Rumaja , 2017

\section{Figura 3}

Corte transversal de raíz del Grupo control

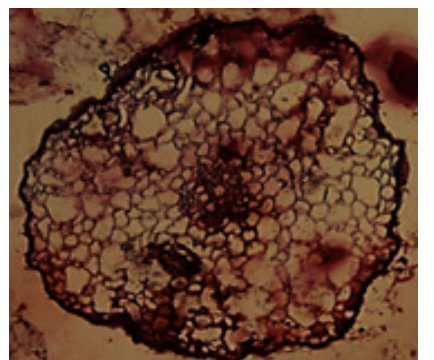

Fuente: Huamán y Rumaja, 2017 
En las figuras 4, 5, 6, 7, 8 y 9 al realizar un análisis comparativo del efecto del mercurio a diferentes concentraciones se puede mencionar que a bajas concentraciones ( 0.1 y $0.5 \mathrm{ppm}$ de mercurio) la especie vegetal $\mathrm{H}$. ranunculoides no muestra cambios en la morfología interna, demostrando así su capacidad de tolerancia y adaptación frente al mercurio.

\section{Figura 4}

Corte trasversal de hoja - Concentración 0.1 ppm.

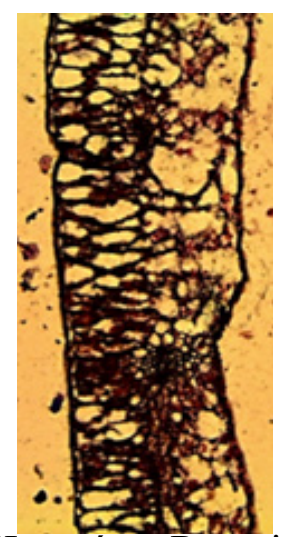

Fuente: Huamán y Rumaja, 2017

\section{Figura 5}

Corte transversal de tallo - Concentración 0.1 ppm.

Fuente: Huaman y Rumaja, 2017

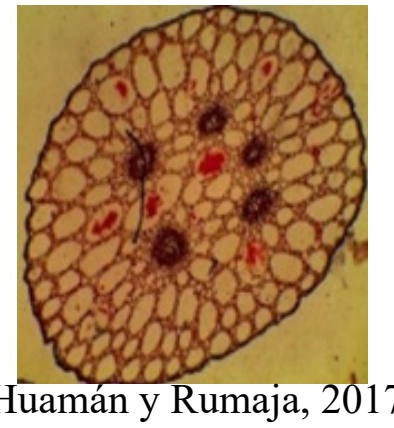

\section{Figura 6}

Corte transversal de raíz - Concentración 0.1 ppm.

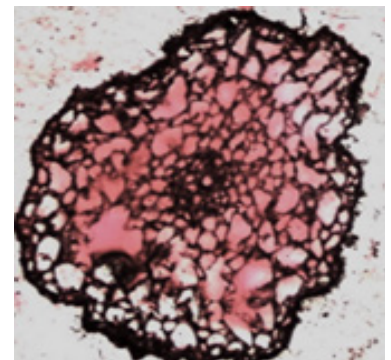

Fuente: Huamán y Rumaja, 2017 


\section{Figura 7}

Corte trasversal de hoja - Concentración 0.5 ppm.

Fuente: Huamán y Rumaja, 2017

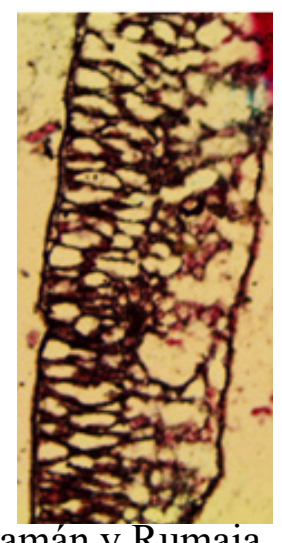

\section{Figura 8}

Corte trasversal del tallo - Concentración 0.5 ppm

Fuente: Huamán y Rumaja, 2017

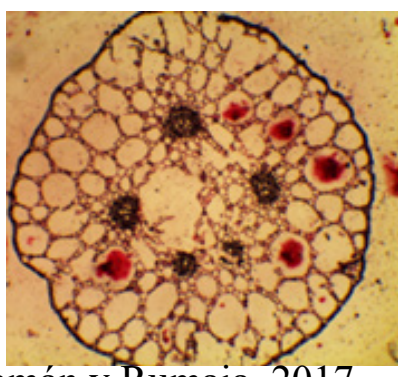

\section{Figura 9}

Corte trasversal de la raíz - Concentración 0.5 ppm.

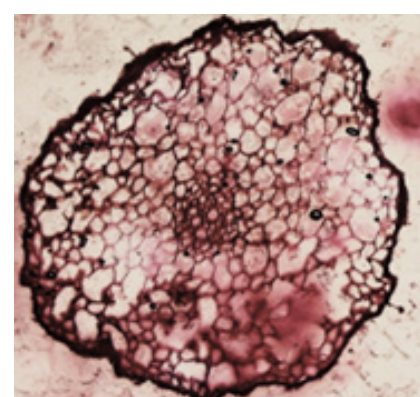

Fuente: Huamán y Rumaja, 2017

En las figuras 10, 11, 12, 13, 14 y 15; a las concentraciones de 1.0 y 2.0 ppm $\mathrm{g} 2+$, se observa que el mercurio provoca alteraciones a nivel de pared celular, membrana plasmática, sistema vascular y organización de los tejidos, en raíz y parte aérea.

De igual modo, a partir de la concentración 1.0 ppm $\mathrm{Hg} 2+$ se observa una indiferenciación de estratos debido a la inhibición del crecimiento celular. 


\section{Figura 10}

Corte trasversal de tallo-Concentración 1.0 ppm

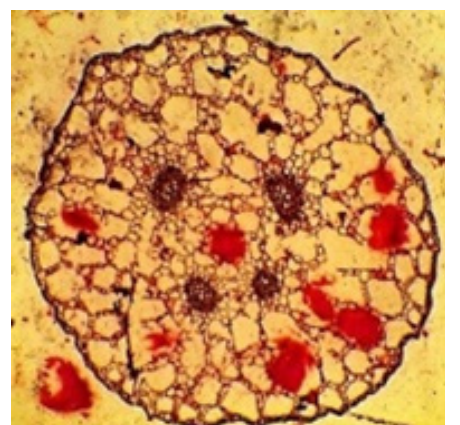

Fuente: Huamán y Rumaja, 2017

\section{Figura 11}

Corte trasversal de hoja - Concentración 1.0 ppm

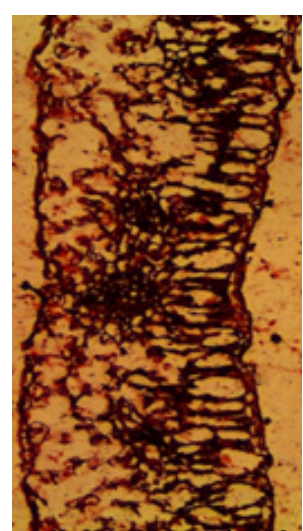

Fuente: Huaman y Rumaja, 2017

\section{Figura 12}

Corte trasversal de raíz - Concentración 1.0 ppm.

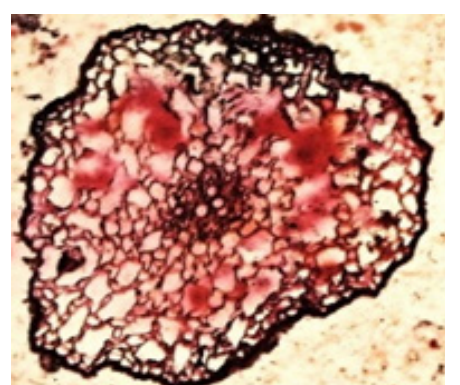

Fuente: Huamán y Rumaja, 2017 


\section{Figura 13}

Corte trasversal de hoja - Concentración 2.0 ppm.

Fuente: Huaman y Rumaja, 2017

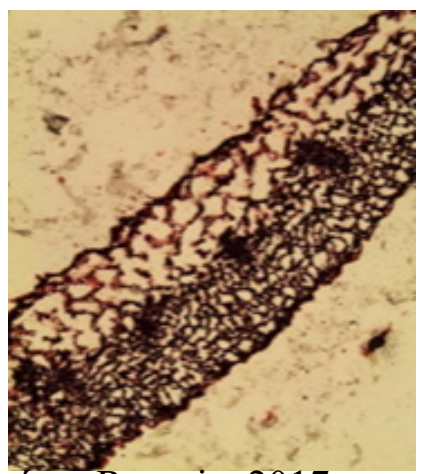

Figura 14

Corte trasversal de tallo - Concentración 2.0 ppm.

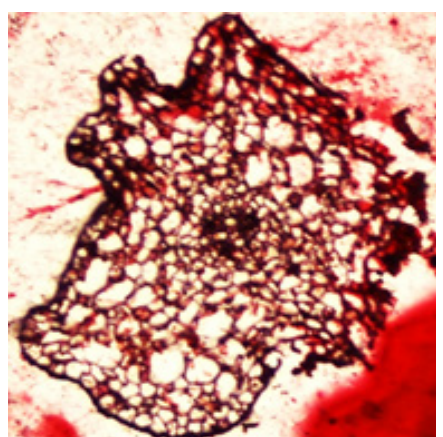

Fuente: Huamán y Rumaja, 2017

\section{Figura 15}

Corte trasversal de raíz - Concentración 2.0 ppm

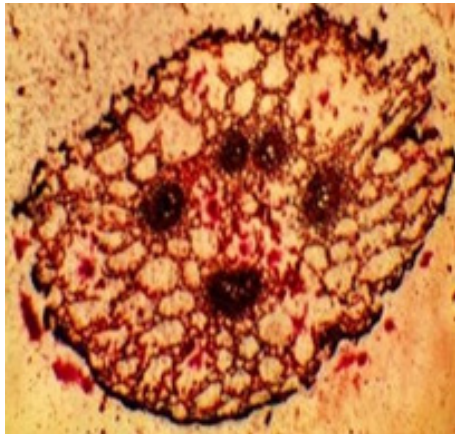

Fuente: Huamán y Rumaja, 2017

En la imagen 16,17 y 18 se observa que tanto para la hoja, tallo y raíz, los daños provocados por el mercurio son más severos, produciendo la necrosis celular. 


\section{Figura 16}

Corte trasversal de hoja - Concentración 5.0 ppm.

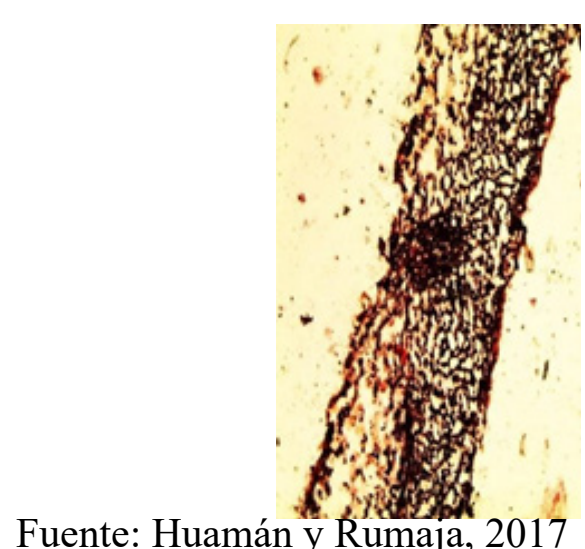

\section{Figura 17}

Corte trasversal de tallo - Concentración 5.0 ppm.

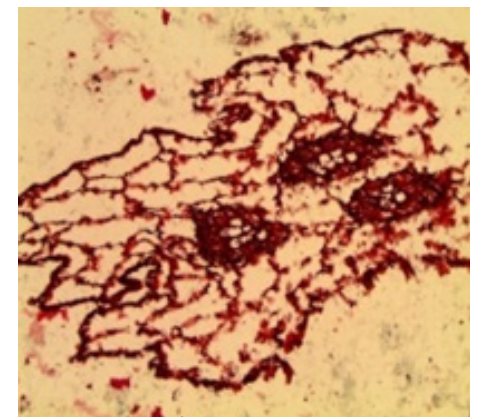

Fuente: Huamán y Rumaja, 2017

\section{Figura 18}

Corte trasversal de raíz - Concentración 5.0 ppm.

Fuente: Huamán y Rumaja, 2017

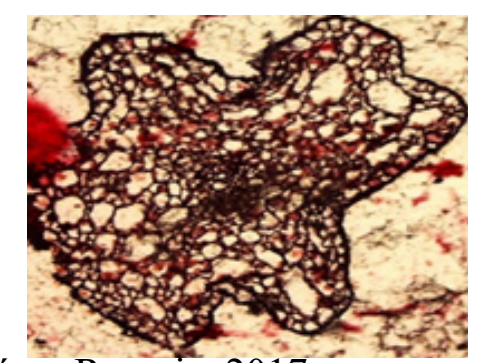


Evaluación de la presencia de clorosis y necrosis en raíz y parte aérea mediante la observación de la especie vegetal Hydrocotyle ranunculoides durante la etapa de contaminación con mercurio

En la tabla 4, los resultados muestran que, la clorosis en estas especies vegetales se debe por efecto del $\mathrm{Hg} 2+$ en el proceso de la fotosíntesis. A mayores concentraciones los daños son más severos produciendo la necrosis celular.

Tabla 4

Clorosis y necrosis de las especies vegetales

DIA Hydrocotyle ranunculoides (Mateccllo)

\begin{tabular}{|c|c|c|c|c|c|c|c|c|c|c|c|}
\hline & \multicolumn{6}{|c|}{ Clorosig } & \multicolumn{5}{|c|}{ Necrosig } \\
\hline & CONT & $\begin{array}{ll}R & 0.1\end{array}$ & 0.5 & 1.0 & 2.0 & 5.0 & 0.1 & 0.5 & 1.0 & 2.0 & 5.0 \\
\hline & $\mathrm{OL}$ & $\mathrm{ppm}$ & $\mathrm{ppm}$ & $\mathrm{ppm}$ & $\mathrm{ppm}$ & $\mathrm{ppm}$ & ppm & ppm & ppm & $\mathrm{ppm}$ & ppm \\
\hline 1 & - & - & - & - & - & - & - & - & - & - & - \\
\hline 2 & - & - & - & - & - & - & - & - & - & - & - \\
\hline 3 & - & - & - & - & - & - & - & - & - & - & - \\
\hline 4 & - & - & - & - & - & - & - & - & - & - & - \\
\hline 5 & - & - & - & - & - & - & - & - & - & - & - \\
\hline 6 & - & - & - & - & - & - & - & - & - & - & + \\
\hline 7 & - & - & - & - & - & - & - & - & - & + & + \\
\hline 8 & - & - & - & + & ++ & ++ & - & - & + & ++ & ++ \\
\hline 9 & - & - & - & + & ++ & ++ & - & - & + & ++ & ++ \\
\hline 10 & - & - & - & + & ++ & ++ & - & - & + & ++ & ++ \\
\hline 11 & - & - & - & + & ++ & ++ & - & - & + & ++ & +++ \\
\hline 12 & - & - & - & + & ++ & ++ & - & - & + & ++ & +++ \\
\hline 13 & - & - & - & + & ++ & ++ & - & - & + & ++ & +++ \\
\hline 14 & - & - & - & + & ++ & ++ & - & - & + & ++ & +++ \\
\hline 15 & - & - & - & + & ++ & ++ & - & - & + & ++ & +++ \\
\hline \\
\hline+++ & \multicolumn{2}{|c|}{ Alta } & \multicolumn{2}{|l|}{+} & \multicolumn{2}{|c|}{ Leve } & & & & & \\
\hline++ & \multicolumn{2}{|c|}{ Moderada } & \multicolumn{2}{|l|}{ - } & \multicolumn{2}{|c|}{ Ausencia } & & & & & \\
\hline
\end{tabular}

\section{Evaluación de la morfometría}

En la tabla 5, se aprecian los síntomas visuales no específicos producidos por el $\mathrm{Hg} 2+$, como son: inhibición del crecimiento radicular y reducción del área foliar. 


\section{Tabla 5}

Evaluación de la morfometría de la especie vegetal Hydrocotyle ranunculoides antes y después de la contaminación con mercurio

Hydrocotyle ranunculoides (mateccllo)

\begin{tabular}{|c|c|c|c|c|c|c|c|}
\hline \multirow{3}{*}{$\begin{array}{c}\text { Parámetr } \\
0\end{array}$} & & \multirow{3}{*}{$\begin{array}{l}\text { Grupo } \\
\text { control } \\
\text { (cm) } \\
\text { 'media' } \\
{ }^{*}{ }^{+} \mathrm{ds}\end{array}$} & \multirow{3}{*}{$\begin{array}{l}\text { Grupo i } \\
\text { (0.1 ppm) } \\
\text { (cm) } \\
\text { "media/ } \\
{ }^{* \star d s}\end{array}$} & \multirow{3}{*}{$\begin{array}{c}\text { Grupo ii } \\
(0.5 \mathrm{ppm}) \\
(\mathrm{cm}) \\
{ }^{+} \text {media/ } \\
t \neq \mathrm{ds}\end{array}$} & \multirow[b]{2}{*}{$\begin{array}{l}\text { Grupo iii }(1.0 \\
\text { ppm) }(\mathrm{cm})\end{array}$} & \multirow[b]{2}{*}{$\begin{array}{c}\text { Grupo iv (2.0 } \\
\text { ppm) (cm) }\end{array}$} & \multirow[b]{2}{*}{$\begin{array}{l}\text { Grupo v } \\
(5.0 \mathrm{ppm}) \\
(\mathrm{cm})\end{array}$} \\
\hline & $\begin{array}{l}\text { Medición antes de la } \\
\text { contaminación }(\mathrm{cm})\end{array}$ & & & & & & \\
\hline & ${ }^{t}$ media/ trds & & & & ${ }^{*}$ medial ${ }^{\star \star} \mathrm{ds}$ & ${ }^{\star}$ media/ ${ }^{* \star} \mathrm{ds}$ & ${ }_{t \rightarrow \pm d s}^{t m e d i a}$ \\
\hline $\begin{array}{l}\text { Talla de la } \\
\text { plantz }\end{array}$ & $13.14 /=0.05$ & $\begin{array}{c}15.711= \\
0.03\end{array}$ & $\begin{array}{c}15.58 / \pm \\
0.05\end{array}$ & $\begin{array}{c}15.447= \\
0.03\end{array}$ & $15.22 / \pm 0.03$ & $14.48 / \pm 0.05$ & $\begin{array}{c}13.921 \pm \\
0.02\end{array}$ \\
\hline $\begin{array}{l}\text { Longitud } \\
\text { del tallo }\end{array}$ & 9.13 & $\begin{array}{c}11.66 /= \\
0.03\end{array}$ & $\begin{array}{c}11.67 /= \\
0.05\end{array}$ & $\begin{array}{c}11.59 /= \\
0.02\end{array}$ & $11.28 /=0.05$ & $11.04 / \pm 0.08$ & $\begin{array}{c}9.41 / \pm \\
0.10\end{array}$ \\
\hline $\begin{array}{l}\text { Diánetro } \\
\text { del tallo }\end{array}$ & $1.28: \pm 0.03$ & $\begin{array}{c}1.66 /= \\
0.01\end{array}$ & $\begin{array}{c}1.64 /= \\
0.04\end{array}$ & $\begin{array}{c}1.64= \\
0.03\end{array}$ & $1.61 / \pm 0.02$ & $1.55 /=0.04$ & $\begin{array}{c}1.43 / \pm \\
0.04\end{array}$ \\
\hline $\begin{array}{l}\text { Longitud } \\
\text { de raz }\end{array}$ & $4.01 i=0.05$ & $\begin{array}{c}9.09 i= \\
0.02\end{array}$ & $\begin{array}{c}9.08 /= \\
0.05\end{array}$ & $\begin{array}{c}9.10 /= \\
0.03\end{array}$ & $8.94 / \pm 0.08$ & $8.57 /=0.05$ & $\begin{array}{c}4.50 / \pm \\
0.05\end{array}$ \\
\hline $\begin{array}{l}\text { Longitud } \\
\text { de hojes }\end{array}$ & $2.11 \neq \pm, 0.06$ & $\begin{array}{c}3.04 /= \\
0.06\end{array}$ & $\begin{array}{c}3.03 /= \\
0.04\end{array}$ & $\begin{array}{c}3.001= \\
0.03\end{array}$ & $2.98 / \pm 0.05$ & $2.71 /=0.04$ & $\begin{array}{c}224 / \pm \\
0.09\end{array}$ \\
\hline $\begin{array}{l}\text { Ancho de } \\
\text { hojas }\end{array}$ & $2.48 i=0.05$ & $\begin{array}{c}2.23 /= \\
0.02\end{array}$ & $\begin{array}{c}3.23 /= \\
0.02\end{array}$ & $\begin{array}{c}3.21 .1 \pm \\
0.02\end{array}$ & $3.16 / \pm 0.02$ & $2.97 / \pm 0.03$ & $\begin{array}{c}258 / \pm \\
0.04 \\
\end{array}$ \\
\hline
\end{tabular}

* Promedio de 3 muestras ** Desviación Estándar

\section{Evaluación del índice y densidad estomática}

En la tabla 6, se observan los resultados del índice y densidad estomática cuantificados en ambas caras (adaxial y abaxial), ya que estas especies son anfiestomáticas. En esta se observa que a medida que incrementan las concentraciones de $\mathrm{Hg} 2+$, ambos factores disminuyen frente al grupo control. 


\section{Tabla 6}

Determinación del índice y densidad estomática de Hydrocotyle ranunculoides (Mateccllo)

\begin{tabular}{|c|c|c|c|c|}
\hline $\begin{array}{l}\text { DE } \\
\text { MERCURI }\end{array}$ & \multicolumn{4}{|c|}{ Hydrocotyle ranunculoides (Mateccllo) } \\
\hline (ppm) & $\begin{array}{c}\text { Índice estomático } \\
\text { Adaxial (\%) }\end{array}$ & $\begin{array}{l}\text { Densidad } \\
\text { estomática } \\
\text { Adaxial }\end{array}$ & $\begin{array}{c}\text { Índice estomático } \\
\text { Abaxial ( } \%)\end{array}$ & $\begin{array}{l}\text { Densidad } \\
\text { estomática } \\
\text { Abaxial }\end{array}$ \\
\hline Control & 17.2 & $\begin{array}{l}1416.298 / 294.199 \\
\text { estomas } / \mathrm{mm}^{2}\end{array}$ & 16.4 & $\begin{array}{l}1357.46 / 266.30 \\
\text { estomas } / \mathrm{mm}^{2}\end{array}$ \\
\hline 0.1 & 17.16 & $\begin{array}{l}1403.867 / 290.884 \\
\text { estomas } / \mathrm{mm}^{2}\end{array}$ & 16.3 & $\begin{array}{l}1344.75 / 261.88 \\
\text { estomas } / \mathrm{mm}^{2}\end{array}$ \\
\hline 0.5 & 17.07 & $\begin{array}{l}1368.508 / 281.768 \\
\text { estomas } / \mathrm{mm}^{2}\end{array}$ & 16.24 & $\begin{array}{l}1306.63 / 253.32 \\
\text { estomas } / \mathrm{mm}^{2}\end{array}$ \\
\hline 1 & 16.91 & $\begin{array}{l}1308.564 / 266.298 \\
\text { estomas } / \mathrm{mm}^{2}\end{array}$ & 16.03 & $\begin{array}{l}1270.99 / 242.54 \\
\text { estomas } / \mathrm{mm}^{2}\end{array}$ \\
\hline 2 & 16.58 & $\begin{array}{l}1293.923 / 257.182 \\
\text { estomas } / \mathrm{mm}^{2}\end{array}$ & 15.25 & $\begin{array}{l}1221.82 / 219.89 \\
\text { estomas } / \mathrm{mm}^{2}\end{array}$ \\
\hline 5 & 16.04 & $\begin{array}{l}1267.956 / 242.265 \\
\text { estomas } / \mathrm{mm}^{2}\end{array}$ & 14.78 & $\begin{array}{l}1211.88 / 210.22 \\
\text { estomas } / \mathrm{mm}^{2}\end{array}$ \\
\hline
\end{tabular}

Evaluación del análisis proximal de la especie vegetal Hydrocotyle ranunculoides antes de la contaminación con mercurio

En la tabla 7, se presentan los valores del análisis proximal realizado antes de la etapa de contaminación con mercurio, los cuales corresponden a la media obtenida del análisis de 3 mediciones. Estos valores están expresados por $1 \mathrm{~g}$ de muestra fresca. En la composición química de esta especie vegetal se observa que la parte aérea presenta un mayor porcentaje de humedad, cenizas, proteínas, grasas y fibra en relación a la raíz. 
Tabla 7

Análisis proximal de Hydrocotyle ranunculoides

Número de Muestras Hydrocotyle ranunculoides (Mateccllo)

\begin{tabular}{|c|c|c|c|}
\hline \multirow{7}{*}{ 罗 } & Parámetro (\%) & & *Media /*\$SD \\
\hline & Humedad & 3 & $94.824 / \pm 0.003$ \\
\hline & Cenizas & 3 & $1.204 / \pm 0.014$ \\
\hline & Proteinas & 3 & $1.230 / \pm 0.010$ \\
\hline & Grasas & 3 & $0.120 / \pm 0.010$ \\
\hline & Fibra & 3 & $0.108 / \pm 0.003$ \\
\hline & Carbohidratos & 3 & $2.514 / \pm 0.019$ \\
\hline \multirow{6}{*}{ 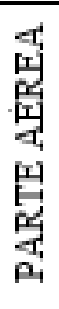 } & Humedad & 3 & $93.700 / \pm 0.010$ \\
\hline & Cenizas & 3 & $1.457 / \pm 0.006$ \\
\hline & Proteinas & 3 & $1.963 / \pm 0.006$ \\
\hline & Grasas & 3 & $0.144 / \pm 0.007$ \\
\hline & Fibra & 3 & $0.119 / \pm 0.002$ \\
\hline & Carbohidratos & 3 & $2.615 / \pm 0.011$ \\
\hline
\end{tabular}

* Promedio de 3 muestras ** Desviación Estándar

\section{Evaluación del análisis proximal de la especie vegetal Hydrocotyle ranunculoides después de la contaminación con mercurio}

En la tabla 8, se muestran los resultados del análisis proximal de $H$. ranunculoides después de la contaminación con mercurio, donde se observa una disminución en cuanto al porcentaje de humedad, cenizas, proteínas, grasas, fibra y un incremento en el porcentaje de carbohidratos.

A las concentraciones de 0.1 y $0.5 \mathrm{ppm} \mathrm{Hg} 2+$ los porcentajes de humedad, ceniza, proteínas, grasas, fibra en raíz y parte aérea disminuyeron y los porcentajes de carbohidratos incrementaron ambos ligeramente respecto al grupo control. Por lo que podemos decir que, a estas concentraciones el $\mathrm{Hg} 2+$ no provoca cambios significativos en la planta, demostrando así una capacidad de tolerancia y adaptación a estas concentraciones.

A las concentraciones $1.0,2.0$ y $5.0 \mathrm{ppm} \mathrm{Hg} 2+$ se muestra una elevada disminución en el porcentaje de humedad en raíz. En la parte aérea al igual que en la raíz se presenta una disminución del porcentaje de humedad con respecto al grupo control.

Para el porcentaje de cenizas se muestra una ligera disminución tanto en la raíz como en la parte aérea.

En el caso del porcentaje de proteínas los resultados muestran una disminución a medida que se incrementa la concentración de $\mathrm{Hg} 2+$.

Para el porcentaje de grasas, los resultados muestran un decremento tanto en la raíz como en la parte aérea a las diferentes concentraciones de $\mathrm{Hg} 2+$.

En el porcentaje de carbohidratos, los resultados muestran un incremento tanto 
en la raíz como en la parte aérea a las diferentes concentraciones de $\mathrm{Hg} 2+$.

\section{Tabla 8}

Análisis proximal de Hydrocotyle ranunculoides (Mateccllo) después de la contaminación con mercurio






\section{Discusiones}

De la concentración final de mercurio en raíz y parte aérea (hojas y tallo) se plantea que las raíces absorben cantidades significativas de metales, pero simultáneamente restringen su traslocación a la parte aérea, ello probablemente debido a que la retención del metal en las raíces se basa en la unión de metal a sitios de intercambio de iones sobre la pared celular y en la precipitación extracelular en la forma de carbonato de mercurio (Torres, 2018; Lane \& Martin, 1977). Los resultados muestran que hay traslocación aún en concentraciones altas, y que la fitotoxicidad es mayor debido a la alta concentración de mercurio, esto podría ser sustentado por el estudio morfológico, donde se observa que a nivel radicular hay alteraciones en el cilindro cortical y Banda de Caspari, los cuales cumplen la función de ser membranas selectivas que al ser destruidas permiten el paso del $\mathrm{Hg} 2+$ hacia la parte aérea

Entonces puede inferirse que, sobrepasado un cierto umbral de concentración de metales, el metabolismo de la especie vegetal se ve comprometido y es incapaz de adaptarse a la acumulación de elementos tóxicos. Además, algunos de los síntomas que presentaron (clorosis y necrosis) son característicos de muerte celular. Sin embargo, en condiciones de estrés moderado se detectaron algunas respuestas específicas a $\mathrm{Hg} 2+$, lo que ayudará a comprender las diferentes respuestas homeostáticas a elementos tóxicos (Torres, 2018; Lane \& Martin, 1977). Por lo que los factores de traslocación en los resultados son mayores a concentraciones 0.1 y $0.5 \mathrm{ppm} \mathrm{Hg} 2+$, con lo que podemos corroborar que a bajas concentraciones el mercurio se trasloca en mayor proporción hasta la parte aérea (Mendieta \& Taisigue, 2014).

Las alteraciones a nivel de pared celular, membrana plasmática, sistema vascular y organización de los tejidos, en raíz y parte aérea, se podría explicar que el $\mathrm{Hg} 2+$ a nivel de la pared celular provoca trastornos enzimáticos en la biosíntesis de sus constituyentes, debido a cambios en el ensamblaje de los polímeros, disposición de los microtúbulos o al aumento de la adhesión celular de la lámina media (Azcon y Talon, 2008; Lindberg \& Wingstrand, 1985).

Según Navarro J. y col., a nivel de la membrana plasmática el $\mathrm{Hg} 2+$ provoca daños mediante diferentes mecanismos como: oxidación y entrecruzamiento de proteínas (grupo tiol), inhibición de las proteínas de membrana que son esenciales como por ejemplo la $\mathrm{H}+$ - ATPasa, cambios en la permeabilidad, composición y fluidez de los lípidos de membrana. $\mathrm{E} 1 \mathrm{Hg} 2+$ sigue esencialmente la vía de la xilema y en sus relaciones con las células vecinas pueden inducir cambios en la pauta de diferenciación del propio sistema vascular (Navarro, Aguilar \& López, 2007).

La clorosis ocurre porque este metal produce alteraciones en los cloroplastos, disminución en la tasa fotosintética, distorsión de la ultra estructura de los cloroplastos, restricción de síntesis de clorofila, plastoquinona y carotenoides, así como también obstrucción del transporte de electrones, inhibición de actividades enzimáticas del ciclo de Calvin y deficiencia del CO2 como un resultado del cierre estomático (Posada y Arroyave 2006; Drazkiewicz, 1994). El Hg2+ perjudica también la absorción de elementos esenciales tales como hierro y magnesio en plantas 
de pepino y como consecuencia inhibe la síntesis de clorofila (Burzynski, 1987).

Estos daños de los aparatos fotosintéticos se deben a la afinidad del $\mathrm{Hg} 2+$ por ligandos de proteínas N- y S-. Asimismo, se ha observado degradación de la clorofila debido al incremento de la actividad clorofilasa (Prieto et al, 2009) (Drazkiewicz, 1994).

En una fase más avanzada de alteración se producen intensos cambios metabólicos y de regulación celular, y ocurre finalmente el estímulo de la senescencia por acumulación crónica del metal pesado (García, 2006).

Los metales pesados al ingresar en las plantas pueden alterar diversos procesos metabólicos trayendo como consecuencia variadas respuestas biológicas a nivel bioquímico, celular y fisiológico manifestándose en el crecimiento y desarrollo de las plantas (Zhongjin \& Neumann, 2000).

La inhibición del crecimiento radicular de estas especies vegetales podría deberse a la inhibición de la proliferación celular y la muerte de las células de los meristemos apicales, así como a la alteración de la permeabilidad de las membranas celulares, por la unión de los iones de $\mathrm{Hg} 2+$ a grupos sulfidrilos de proteínas y a la parte hidrofílica de fosfolípidos, afectando la homeostasis iónica y la subsecuente alteración de la actividad de enzimas cruciales en el metabolismo de las plantas (Paz, 2014; Janicka et al, 2008).

Estos síntomas se evidencian más a medida que las concentraciones de mercurio incrementan, por ejemplo, la disminución del crecimiento se hace más evidente a concentraciones de $1.0,2.0$ y $5.0 \mathrm{ppm}$ para $\mathrm{H}$. ranunculoides. La longitud de las raíces, tallos y hojas de $\mathrm{H}$. ranunculoides disminuyen significativamente después de ser contaminadas con $\mathrm{Hg} 2+$ a las concentraciones de $1.0,2.0$ y 5.0 ppm $\mathrm{Hg} 2+$ durante 15 días respecto al grupo control. Esta tendencia también ha sido observada en raíces de Pisum sativum y Mentha spicata cultivadas con $5000 \mathrm{ug} / \mathrm{L}$ de HgCl2 (Barceló J. \& Poschenrieder Ch., 1992) y en el desarrollo y crecimiento de Taraxacum officinale a las concentraciones de 50, 100, $150 \mathrm{ppm}$ de plomo (Alba, 2013).

Bethke y Drew mencionan que, la transpiración y la intensidad de la respiración están en razón directa al número y abertura de los estomas que influyen directamente sobre la asimilación clorofílica, señalando que la disminución de la cantidad de estomas por $\mathrm{mm} 2$ incrementa la resistencia estomática de la planta y de esta manera evita un exceso de transpiración; sin embargo, tanto la densidad estomática (DE) como el índice estomático (IE) son tan variables que están fuertemente influenciadas por diversas condiciones estresantes como condiciones de sequía y altas concentraciones salinas (Salas, Sanabria y Pire, 2001)(Bethke \& Drew, 1999).

La deshidratación se debe al estrés oxidativo inducido por el $\mathrm{Hg} 2+$, lo que produce una inhibición en los canales de agua y la disminución de la conductividad hidráulica desde la raíz hasta las hojas limitando el crecimiento de las raíces, como refieren Zhongiin Lu y Neumann. Se ha demostrado también que los iones de $\mathrm{Hg} 2+$ inhiben rápidamente el transporte de agua a través de las raíces aisladas de plantas de cereales como maíz y trigo (Zhongjin \& Neumann, 2000; Zhang, Stephen, \& Tyerman, 2000). 
El Hg2+ altera los niveles de elementos minerales debido a que bloquea la entrada de cationes $(\mathrm{K}+, \mathrm{Ca} 2+, \mathrm{Mg} 2+, \mathrm{Mn} 2+, \mathrm{Zn} 2+, \mathrm{Cu} 2+, \mathrm{Fe} 3+)$ y aniones $(\mathrm{NO} 3-)$ en el sitio de absorción de las raíces, esto se relacionada con el tamaño de los radios iónicos del metal (Posada y Arroyave, 2006; Hernández, 2002; Douglas, 1991).

La disminución de proteínas se puede explicar debido a que el $\mathrm{Hg} 2+$ forma mercáptidos con el grupo (-SH) de la cisteína, complejos con los grupos fosfatos e incremento de la actividad enzimática (proteasas), provocando así una acelerada hidrólisis de proteínas, esto según Jana y Choudhuri. Al igual que la raíz, la parte aérea muestra una disminución de $0.013,0.025,0.137,0.193$ y $0.227 \%$ en relación al grupo control, esto debido a los mismos mecanismos de fitotoxicidad que ocurren en la raíz (Prieto et al, 2009; Jana \& Choudhuri, 1982).

El $\mathrm{Hg} 2+$ altera la proporción de lípidos membranales en el tejido foliar, observándose una marcada reducción en el contenido de los glucolípidos Monogalactosil Diacilglicerol (MGDG) y Digalactosil Diacilglicerol (DGDG), así como un incremento de los fosfolípidos Fosfatidilcolina (FC) y Fosfatidiletanolamina (FE). A nivel de raíz, se observa una reducción en el contenido de esteroles totales, principalmente el sitosterol, sin embargo, el contenido de estigmasterol presenta un incremento. Adicionalmente, se presenta una disminución en el contenido de FE y FC. De forma general, el Hg2+ causa una reducción en el contenido de lípidos totales (Alonso, 2011; Jana \& Choudhuri, 1982).

El incremento de azúcar se puede explicar a que este produce una disminución en el transporte de sacarosa, debido a que el $\mathrm{Hg} 2+$ se une fuertemente a los grupos carboxilo de los carbohidratos del ácido galacturónico y del ácido glucorónico en la pared celular, lo cual restringe su transporte vía apoplasto, por lo tanto, provoca una acumulación en hojas y raíces (Devi, Nidhi, Anil, 2012; García, 2006).

Asimismo, Devi R. halló un aumento del 10-20\% en el contenido de sacarosa al cabo de siete días en plantas de guisante (Pisum sativum L.). La explicación de estas respuestas puede deberse a una serie de mecanismos de defensa no específicos los cuales son activados cuando las plantas son expuestas a contaminación por plomo. Estos incluyen la síntesis de osmolitos, entre los cuales se encuentran la glucosa y la prolina (Devi, Nidhi, Anil, 2012; García, 2006).

\section{Conclusiones}

En el estudio se evaluó la capacidad fitorremediadora de la especie vegetal Hydrocotyle ranunculoides en relación a la contaminación con mercurio, concluyendo que la capacidad fitorremediadora dependerá de la concentración de mercurio que se encuentre en el agua, por lo tanto $\mathrm{H}$. ranunculoides obtuvo un factor de bioacumulación $>1000 \mathrm{mg} / \mathrm{L}$ hasta la concentración $1.0 \mathrm{ppm} \mathrm{Hg} 2+$, mientras que para las concentraciones de 2.0 y $5.0 \mathrm{ppm} \mathrm{Hg} 2+$ es tolerante. El factor de traslocación para las concentraciones: 0.1 y $0.5 \mathrm{ppm} \mathrm{Hg} 2+$ es $>1 \mathrm{mg} / \mathrm{L}$, indicando que el mercurio se encuentra también en la parte aérea, mientras que para las concentraciones 1.0, 2.0 y $5.0 \mathrm{ppm} \mathrm{Hg} 2+$ el mercurio se encuentra en mayor proporción en la raíz, la mayor concentración de mercurio en la raíz se relaciona con el daño que este metal produce en la 
morfología externa (clorosis y necrosis) e interna de la raíz y parte aérea de Hydrocotyle ranunculoides, como alteraciones a nivel de la pared celular y organización de tejidos a medida que la concentración de mercurio incrementa, hasta llegar a una senescencia celular. Esta toxicidad altera también la composición química en la raíz, encontrándose una disminución del porcentaje de humedad en un $1.89 \%$, cenizas $0.23 \%$, proteínas $0.34 \%, 0.06 \%$ grasas y $0.02 \%$ fibra a medida que se incrementa la concentración del mercurio; mientras que el porcentaje de carbohidratos incrementó en un $2.43 \%$, en comparación al grupo control. Mientras que para la parte aérea la disminución en el porcentaje de humedad fue $1.82 \%$, cenizas $0.27 \%$, proteínas $0.844 \%$, grasas $0.07 \%$ y fibra $0.01 \%$ y un incremento de $3.01 \%$ para carbohidratos (Huamán y Rumaja, 2017).

\section{Agradecimientos}

Esta investigación fue apoyada por la Universidad Nacional de San Antonio Abad del Cusco, Perú, a través de un financiamiento otorgado a las Bachilleres Jackeline Zúmiko Huamán Tito y Abigail Rumaja Santos (Consejo de unidades de investigación). Se agradece a la Mgt. Anahí Karina Cardona Rivero, al Ing. Mario Cumpa Cayuri y Mgt. Janet González Bellido por su apoyo y asesoramiento en la realización de pruebas experimentales en los laboratorios y al Blgo. José Luis Sierra Herrera por la ayuda brindada durante el análisis de nuestras muestras en el laboratorio de Farmacognosia y Productos Naturales:

\section{Declaración de conflicto de interés}

Los autores declaran no tener ningún tipo de conflicto de intereses 


\section{Referencias}

Alba B. (2013). Capacidad bioacumuladora de plomo, a diferentes concentraciones, en raíz, tallo y hoja de Taraxacum officinale en condiciones de laboratorio. 1(2): 51 - 60. 2013 recuperado de https://docplayer.es/97800024-Capacidadbioacumuladora-de-plomo-a-diferentes-concentraciones-en-raiz-tallo-y -hoja-de-taraxacum-officinale-en-condiciones-de-laboratorio.html

Alonso J. (2011) Manual de histología vegetal $1^{\circ}$ Edición, México: Editorial Mundi prensa AOAC International, Official methods of analysis of AOAC International. (s. f.). Recuperado de http://www.eoma.aoac.org/

Azcón B., \& Talón M. (2008). Fundamentos de la fisiología vegetal (2.a ed.). Madrid - España: Mcgraw-Hill S.A.

Barceló J., \& Poschenrieder Ch. (1992). Respuestas de las plantas a la contaminación por metales pesados Laboratorio de Fisiología Vegetal. Facultad de Ciencias. Universidad Autónoma de Barcelona. 2, 345-361.

Barrientos, A., \& Borys M. (2003). Índice y densidad estomática en plántulas de tres razas de aguacatero. 26 (4)(Fitotec. Mex), 285-290.

Bethke P. C., \& Drew M. C. (1999). Stomatal and Nonstomatal Components to Inhibition of Photosynthesis in Leaves of Capsicum annum during Progressive Exposure to $\mathrm{NaCl}$ Salinity. 99, 219-226.

Burzynski M. (1987). Influence of lead and cadmium on the absorption and distribution of potassium, calcium, magnesium and iron in cucumber seedlings. 9, 229-238.

Cabo S. M., \& Frers C. (2008). El uso de plantas acuáticas para el tratamiento de aguas residuales. Observatorio Medioambiental, Universidad Complutense de Madrid España (UCM). 11, 301-305.

Cano Vera Mirian J. (1999). Análisis bromatológico nutricional de nueve especies de alfalfa Cusco. Universidad Nacional San Antonio Abad del Cusco, Carrera profesional de Química.

Cubero D., \& Vieira M. (1999). Abonos orgánicos y fertilizantes químicos, son compatibles con la agricultura. Conferencia 77: Manejo de la nutrición y fertilización del cultivo del café orgánico. Costa Rica. 1, 61-67.

Delgadillo A., González C., García F., Villagómez J., Acevedo O., (2011). Evaluación de la bioacumulación y toxicidad de cadmio y mercurio en pasto llanero (Brachiaria dictyoneura). 14 (2011): 597- 612 Recuperado de http://www.scielo. org.mx/pdf/tsa/v14n2/v14n2a2.pdf 
Devi R., Nidhi M., Anil K., (2012) Effect of exogenous lead on growth and carbon metabolism of pea (Pisum sativum L) seedlings, de la revista Springer link 19, pp 81-89 Recuperado de http://link.springer.com/article/10.1007/s12298-012 $-0143-5$

Díaz F. (2016). Mercurio en la minería del oro: impacto en las fuentes hídricas destinadas para consumo humano. 16 (6): 947-957 Recuperado de http://www. scielo.org.co/pdf/rsap/v16n6/v16n6a12.pdf

Douglas L. (1991). Lead Influences Root Growth and Mineral Nutrition of Picea abies Seedlings. 139, 95-99.

Drazkiewicz M. (1994). Chlorophyllase: Occurrence, functions, mechanism of action, effects of external and internal factors. Agris. 30, 321-331.

Eaton A., Cresceri L.y Greenberg A. (1995). Standards methods for the examination of water and wastewater, APHA-AWWA-WEF, 19a edition Method 3500- Hg C, page. 3-79.

Foy C., Chaney R. L., \& White M C. (2000). The Physiology of Metal Toxicity in Plants, Plant physiology. 29. Recuperado de http://www.annualreviews.org/doi/ abs/10.1146/annurev.pp.29.060178.002455

García V. D. (2006). Efectos fisiológicos y compartimentación radicular en plantas de Zea mays L. expuestas a la toxicidad del plomo. Recuperado de http://www. tdx.cat/bitstream/handle/10803/3676/dgv1de1.pdf;jsessionid=EAA6DCC0FD 303F6A4F28164E444E9DB0?sequence $=1$

Gisbert C. A. (2001). Medicina Legal y Toxicología(5.a ed.). España: MASSON S.A.

Hernández R. (2002). Nutrición mineral en plantas. Recuperado de http://www.forest. ula.ve/ rubenhg/nutricionmineral/.

Huamán J., Rumaja A. (2017). Evaluación de la capacidad fitorremediadora de las especies vegetales Nasturtium officinale W. T. Aiton (Berro) e Hydrocotyle ranunculoides L. f. (Mateccllo) en relación a la contaminación con mercurio a diferentes concentraciones (tesis de pre grado).

Jana S. M., \& Choudhuri A. (1982). Senescence in submerged aquatic angiosperms: Effects of heavy metals. 90, 477-484.

Janicka M., Kaba K., Burzynski M., \& Grazyna K. (2008). Response of plasma membrane ATPase to heavy metal stress in Cucumis sativus roots. Experimental Botany. 59, 3721-3728.

Juárez Cahuana R. (2011). Estudio de la composición química y la actividad antioxidante de la pulpa del fruto de Corryocactus ayacuchoensis "Muñununka") Cusco (Tesis de pregrado) Universidad Nacional San Antonio Abad del Cusco. Carrera 
profesional de Química.

Lane S. D., \& Martin E. S. (1977). A Histochemical Investigation of Lead Uptake in Raphanus sativus, The New Phytologist. 79, 281-286.

Lindberg S., \& Wingstrand G. (1985). Mechanism for $\mathrm{Cd} 2+$ inhibition of $(\mathrm{K}+, \mathrm{Mg} 2+)$ ATPase activity and $\mathrm{K}+(86 \mathrm{Rb}+)$ uptake join roots of sugar beet (Beta vulgaris) -Wingstrand-Physiologia Plantarum. 63. Recuperado de http:// onlinelibrary.wiley.com/doi/10.1111/j.1399-3054.1985.tb01900.x/abstract

Mendieta W. C., \& Taisigue L. K. (2014). Acumulación y traslocación de metales, metaloides y no metales en plantas nativas de la zona minera de Chontales: Implicaciones para el potencial de fitoremediación (Tesis de pre grado) Universidad Nacional Autónoma de Nicaragua, Managua. Recuperado de http:// biorem.univie.ac.at/fileadmin/user_upload/p_biorem/education/research/ methods/Accumulation-and-Translocation-metals-in-native-plants_Extended -abstract.pdf

Navarro J.P., Aguilar A., \& López J.R. (2007). Aspectos bioquímicos y genéticos de la tolerancia y acumulación de metales pesados en plantas. 16 (2), 1-17.

Paz A. A. (2014). Efecto del arsénico sobre el crecimiento y la actividad ascorbato peroxiodasa en Rorippa Nasturtium aquaticum (L.) Hayek. (Universidad Nacional De Trujillo - Escuela Académico Profesional De Ciencias Biológicas). Recuperado de http://dspace.unitru.edu.pe/xmlui/bitstream/handle/UNI TRU/4355/Paz\%20Arqueros\%2c\%20Angelica\%20Mercedes.pdf? sequence $=1 \&$ isAllowed $=\mathrm{y}$

Pinto H. H. (2014). Contaminación ambiental en Tintaya. Universidad Nacional Mayor de San Marcos. Investigaciones sociales Lima-Perú. 18 (33), 201-216.

Posada M., Arroyave M. (2006) Efectos del mercurio sobre algunas plantas acuáticas, de la revista SCIELO (6): 57-67. Recuperado de http://www.scielo.org.co/pdf/ eia/n6/n6a06.pdf

Prieto J., González C., Román A., Prieto F., (2009) Contaminación y fitotoxicidad en plantas por metales pesados provenientes de suelos y agua en la revista Tropical and Subtropical Agroecosystems 10 (2009): 29 - 44 Recuperado de https://www. redalyc.org/pdf/939/93911243003.pdf

Salas J., Sanabria M., Pire R.(2001) Variación en el índice y densidad estomática en plantas de tomate (Lycopersicon esculentum Mill) sometidas a tratamientos salinos en la revista Bioagro 13(3):99-104) Recuperado de https://www.redalyc. org/pdf/939/93911243003.pdf

Sandell E.B. (1950). Colorimetric Determination of Traces of Metals. (2.a ed., Vol. 3). London:INC Interscience Publishers. 
Torres A. (2018) Factor de bioconcentración y traslocación de especies altoandinas para suelos contaminados con metales pesados provenientes de la planta concentradora de Mesapata, en condiciones de invernadero, 2015 - 2016" (Tesis de pre grado) Universidad Nacional Santiago Antúnez De Mayolo Recuperado de http://repositorio.unasam.edu.pe/handle/UNASAM/2110

Zhang W., Stephen D., \& Tyerman. (2000). Inhibition of Water Channels by $\mathrm{HgCl} 2$ in Intact Wheat Root Cells. Plant Physiologists. 120, 849-857.

Zhongjin L., \& Neumann P. (2000). Water Stress Inhibits Hydraulic Conductance and Leaf Growth in Rice Seedlings but Not the Transport of Water via MercurySensitive Water Channels in the Root. 120, 143-151. 